\title{
The puzzling unsolved mysteries of liquid water: Some recent progress
}

\author{
H.E. Stanley, ${ }^{\mathrm{a}, *}$, P. Kumar ${ }^{\mathrm{a}}$, L. Xu ${ }^{\mathrm{a}}$, Z. Yan ${ }^{\mathrm{a}}$, M.G. Mazza ${ }^{\mathrm{a}}$, \\ S.V. Buldyrev ${ }^{\mathrm{a}, \mathrm{b}}$, S.-H. Chen ${ }^{\mathrm{c}}$, F. Mallamace ${ }^{\mathrm{d}}$ \\ ${ }^{a}$ Department of Physics, Center for Polymer Studies, Boston University, Boston, MA 02215, USA \\ ${ }^{\mathrm{b}}$ Department of Physics, Yeshiva University, 500 West 185th Street, New York, NY 10033, USA \\ ${ }^{\mathrm{c}}$ Nuclear Science and Engineering Department, Massachusett Institute of Technology, Cambridge, MA 02139, USA \\ ${ }^{\mathrm{d}}$ Dipartimento di Fisica, Univ. Messina, Vill. S. Agata, C.P. 55, I-98166 Messina, Italy
}

Available online 31 July 2007

\begin{abstract}
Water is perhaps the most ubiquitous, and the most essential, of any molecule on earth. Indeed, it defies the imagination of even the most creative science fiction writer to picture what life would be like without water. Despite decades of research, however, water's puzzling properties are not understood and 63 anomalies that distinguish water from other liquids remain unsolved. We introduce some of these unsolved mysteries, and demonstrate recent progress in solving them. We present evidence from experiments and computer simulations supporting the hypothesis that water displays a special transition point (which is not unlike the "tipping point" immortalized by Malcolm Gladwell). The general idea is that when the liquid is near this "tipping point," it suddenly separates into two distinct liquid phases. This concept of a new critical point is finding application to other liquids as well as water, such as silicon and silica. We also discuss related puzzles, such as the mysterious behavior of water near a protein.
\end{abstract}

(C) 2007 Elsevier B.V. All rights reserved.

Keywords: Liquid-liquid phase transition; Liquid-liquid critical point; Low-density liquid; High-density liquid

\section{Introduction}

Most students think that randomness means uncorrelated randomness. They learn that statistical physics deals solely with random phenomena, so they imagine that our field cannot possibly yield any insights into the real world as they correctly know that no system in which they are interested corresponds to simple uncorrelated randomness. Hence we found using the adjective "correlated" helped persuade our collaborators that what we do may possibly be applicable to systems in which they are interested.

To help educate ourselves, we have learned to present simple visual examples of the concept of correlated randomness. One example we found useful was comparing a simple, unbiased random walk in two dimensions ("uncorrelated randomness") and a simple, self-avoiding random walk in two dimensions ("correlated

\footnotetext{
*Corresponding author. Tel.: + 16173532617 ; fax: + 16173533783.

E-mail address: hes@bu.edu (H.E. Stanley).
} 
randomness"). In the case of the uncorrelated walk, the spread of a $10^{4}$ step path is $10^{2}$. In the correlated random walk, the spread of a $10^{4}$ step path is on the order of $10^{3}$ steps, an order of magnitude larger (Fig. 1).

A second simple example of correlated randomness that people from other fields can appreciate is critical opalescence, first discovered and interpreted - in terms of correlated randomness - by Andrews in 1869 [1]. This can occur in two-component fluids but also in one-component fluids-the so-called liquid-liquid phase transition [2]. In the more traditional two-component fluid, the concentrations of the two components and the temperature have been adjusted so that the system is near its consolute point. The correlated fluctuations observed at that consolute point are so strong that their length scale has become comparable to the wavelength of visible light and one sees a scattering of that visible light in the form of an opalescent glow.

\section{Water}

Water is perhaps the most ubiquitous, and the most essential, of any molecule on earth. Indeed, $\mathrm{H}_{2} \mathrm{O}$ challenges the imagination of even the most creative science fiction writers (such as $\mathrm{K}$. Vonnegut) to picture what life would be like without water, and one often hears the adage "biology cannot be understood until water is understood". Despite 300 years of research, however, the 63 anomalies that distinguish water from other liquids lack a coherent explanation (http://www.lsbu.ac.uk/water/anmlies.html) so sometimes water is called the prototype "complex fluid".

We will introduce some of the 63 anomalies of water, and will demonstrate some recent progress in solving them using concepts borrowed from various disciplines including chemistry and physics. In particular, we will present evidence from experiments designed to test the hypothesis that water displays a special transition point (which is not unlike the "tipping point" immortalized in Malcolm Gladwell's book of the same title). The general idea that when water is near this tipping point, it can separate into two distinct liquid phases distinguished by their density. This new concept of a critical point is also proving useful in understanding some of the anomalies of other liquids, such as silicon, silica, and carbon.

We will also discuss two other water mysteries, such as the puzzling behavior of water near a protein, and the breakdown of the Stokes-Einstein relation in supercooled water.

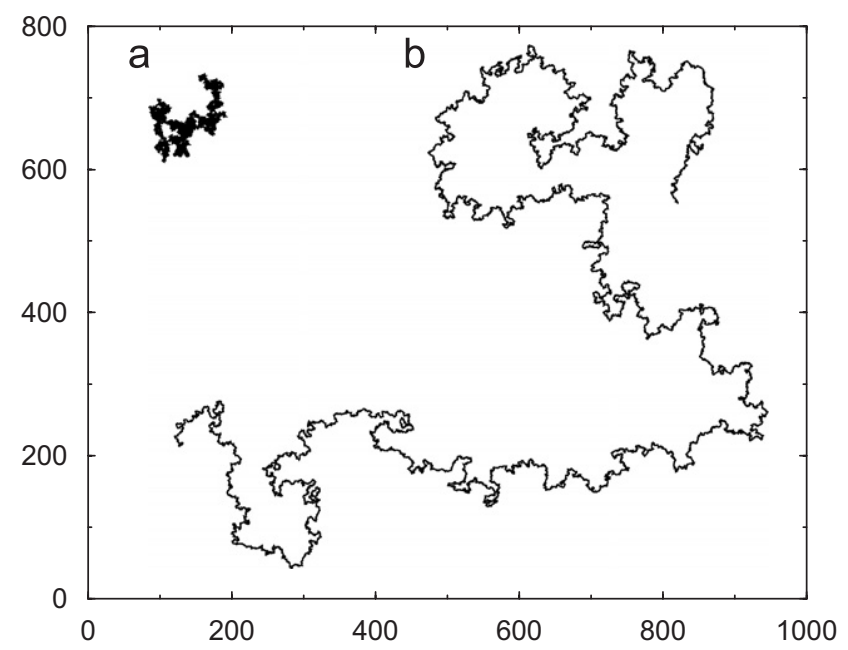

Fig. 1. (a) The trail of a random walk of $10^{4}$ steps, compared with (b) the trail of a self-avoiding random walk of the same number of steps. The "correlated randomness" of the latter results in drastically different behavior. Specifically, the characteristic diameter jumps by a factor of 10 , from approximately $\left(10^{4}\right)^{1 / 2}=100$ to approximately $\left(10^{4}\right)^{3 / 4}=1000$, where we have used the fact that the fractal dimensions (defined as the exponent to which the length is raised to obtain the mass) are 2 and $\frac{4}{3}$, respectively. This figure is courtesy of S.V. Buldyrev. 


\subsection{What is the phenomenon?}

We start with three thermodynamic functions. The first is the compressibility - the response of the volume to an infinitesimal change in pressure. In a typical liquid, this response function decreases when we lower the temperature. I understand this decrease via statistical physics. This thermodynamic response function is proportional to the thermal average of all the fluctuations in specific volume in the system. As we lower the temperature, we imagine that fluctuations of necessity decrease, thus the compressibility decreases.

Water is unusual in three respects. First, the average compressibility of water is twice as large as what one would expect were water a typical fluid and were one to plug all the prefactors into the formulas that give compressibility in terms of volume fluctuations. Second, the magnitude of that factor of two actually increases as one lowers the temperature. That being the case, there is ultimately a minimum-which occurs at $46^{\circ} \mathrm{C}$. Below that temperature, the compressibility increases dramatically. At the lowest attainable temperature $\left(-40^{\circ} \mathrm{C}\right)$ the compressibility takes on a value that is twice of that at the minimum. This is not a tiny effect; it is huge (Fig. 2).

The second thermodynamic function is the specific heat, and we observe three similar anomalies: it is twice as large as that of a typical liquid, the discrepancy gets bigger as the temperature is lowered, and a minimum occurs at $35^{\circ} \mathrm{C}$.

The third thermodynamic function is the coefficient of thermal expansion, the response of the volume to an infinitesimal change in temperature. This quantity we assume to always be positive because if there is a local region of the liquid in which the specific volume is larger than the average, then there will be more arrangements of the molecules and hence the entropy will be larger than the average. This is true of almost all liquids, but the magnitude of this cross-fluctuation of volume and entropy in water is approximately three times smaller than we would expect, and at $4{ }^{\circ} \mathrm{C}$ the coefficient of thermal expansion passes through zero and actually becomes negative.
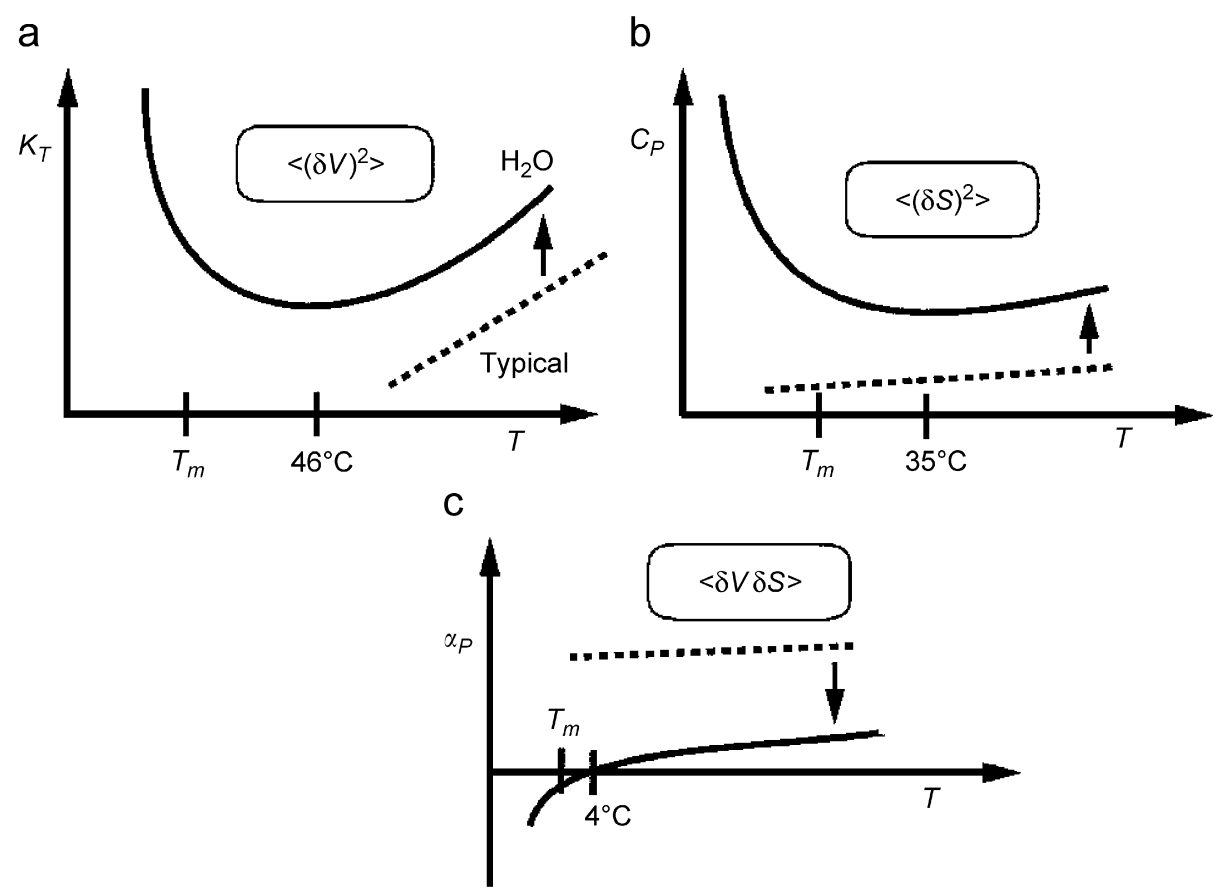

Fig. 2. Schematic dependence on temperature of: (a) the isothermal compressibility $K_{T}$, (b) the constant-pressure specific heat $C_{P}$, and (c) the thermal expansivity $\alpha_{P}$. The behavior of a typical liquid is indicated by the dashed line, which, very roughly, is an extrapolation of the high-temperature behavior of liquid water. Note that while the anomalies displayed by liquid water are apparent above the melting temperature $T_{m}$, they become more striking as one supercools below $T_{m}$. 


\subsection{Why do we care about this anomalous behavior?}

To begin with, if we do not understand water we will never understand biology. That is a major reason to care. Scientifically, water is the prototype complex fluid. It is not a simple, "bag-of-marbles" liquid, but a "bag of tetrahedra." These tetrahedra are not only irregularly shaped, but are charged. Two of the arms are positively charged, corresponding to the protons on each water molecule, and two are negatively charged, corresponding to the lone pairs. In addition to short-range forces, these "charged tetrahedra" interact with long-range Coulomb forces.

\subsection{What do we do?}

Our approach is based on the fact that water has a tetrahedral local geometry. In this sense water shares features with other liquids such as silicon. Because water is both tetrahedral and charged means that a simple Lennard-Jones potential is not sufficient to describe its complexity. One way to modify the Lennard-Jones potential to provide at least a simplified description is to bifurcate the single minimum into two minima [3-6]. The first minimum, at a closer distance, corresponds to two pentamers (a water molecule and its four neighbors) of water interacting with each other in a rotated configuration. The second minimum, at a greater distance, occurs in the unrotated position. This second position is a deeper minimum because although the pentamers are farther apart there is the potential for hydrogen bonding between the molecules and we can see the beginnings of an ice-like hexagonal structure (Fig. 3).

\section{a}

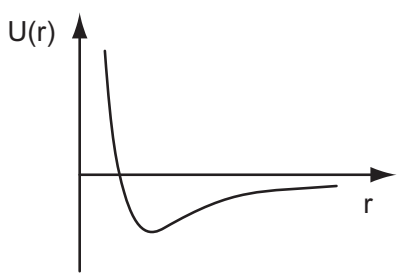

b

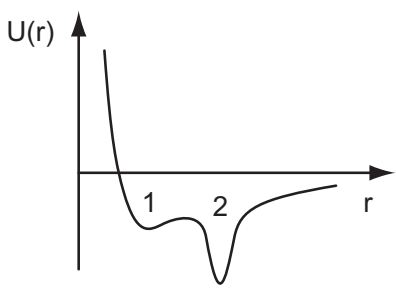

C

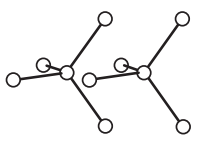

1

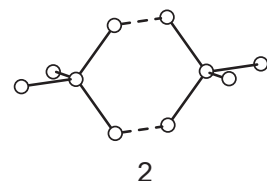

2

Fig. 3. Physical arguments relating to the plausibility of the existence of the known liquid-gas critical point $C$ and the hypothesized LDL-HDL critical point $C^{\prime}$. (a) Idealized system characterized by a pair interaction potential with a single attractive well. At low enough $T\left(T<T_{c}\right)$ and high enough $P\left(P>P_{c}\right)$, the system condenses into the "liquid" well shown. (b) Idealized system characterized by a pair interaction potential whose attractive well has two sub-wells, the outer of which is deeper and narrower. For low enough $T\left(T<T_{c^{\prime}}\right)$ and low enough $P\left(P<P_{c^{\prime}}\right)$, the one-phase liquid can "condense" into the narrow outer "LDL" sub-well, thereby giving rise to a LDL phase, and leaving behind the high-density liquid phase occupying predominantly the inner subwell. (c) Two idealized interaction clusters of water molecules ("Walrafen pentamers") in configurations that may correspond to the two sub-wells of (b). This figure is courtesy of Dr. O. Mishima. 
The important point is that there are two minima with the outer one corresponding to a larger specific volume - because the distance is larger - and a lower entropy. The possibility is that liquid water could at low temperatures condense not into a single phase - as we anticipate when a gas with a simple interaction like a Lennard-Jones potential condenses into a fluid - but into two different phases. This possibility was first raised by Takahashi 60 years ago and various elaborations of this model have been made by a number of people since then, including seminal work of Per Hemmer and George Stell in 1971 [7-9]. The implications of this is the possibility of two different liquid phases contributing to an increase in these fluctuations in specific volume and a negative contribution to the cross-fluctuations, negative because the deeper well has a larger volume and a lower entropy. The consequences of this fact qualitatively explain the phenomenon we were describing at the beginning - volume fluctuations are increased, entropy fluctuations are increased, and cross-fluctuations of volume and entropy are decreased. This picture further predicts the possibility that at low temperatures there will be a genuine phase transition in which the single component liquid separates into two different phases. The implications of this when applied to real water molecules produce a phase diagram of liquid water (Fig. 4). This was first uncovered by Poole et al. [10]. At one atmosphere (the left axis) we see the melting temperature and the limit of supercooling around $-40{ }^{\circ} \mathrm{C}$. At a very low temperature we see the presence of the glassy phase, not unlike that of any other liquid except that at high pressure this glassy phase shifts from a low-density form to a high-density form. In typical liquids we do not find two different glassy phases. These two forms correspond to the two different local arrangements characteristic of water tetrahedra. The order parameter jump between these two phases is not a trivial amount, but on the order of $30 \%$ [11].

Between the liquid and glassy phases of water we have a region in which water does not exist as a liquid. I like to call this a "No Man's Land". The hypothesis that follows from the reasoning we have just described is that this first-order phase transition line known to separate the two amorphous forms of solid water extends into this No Man's Land and ultimately terminates at a critical point. Just as the glassy water first-order transition line separates a low-density amorphous from a high-density amorphous phase of water, so also this extension of the line into the liquid region separates a low-density liquid (LDL) from a high-density liquid (HDL). The power-law behavior uncovered over the years by Angell, Anisimov and collaborators corresponds to the fact that the extension of this first-order line beyond the critical point - the "Widom line"- has the effect where any experiment approaching that line looks as though it is going to diverge with critical exponents but does not.

This phase diagram is hypothesized, but it has not been proved. What has been proved is that computer simulations using tried and tested models of liquid water confirm the broad features of this phase diagram (see Ref. [12] and references therein). But computer models of water (like computer models of anything) are

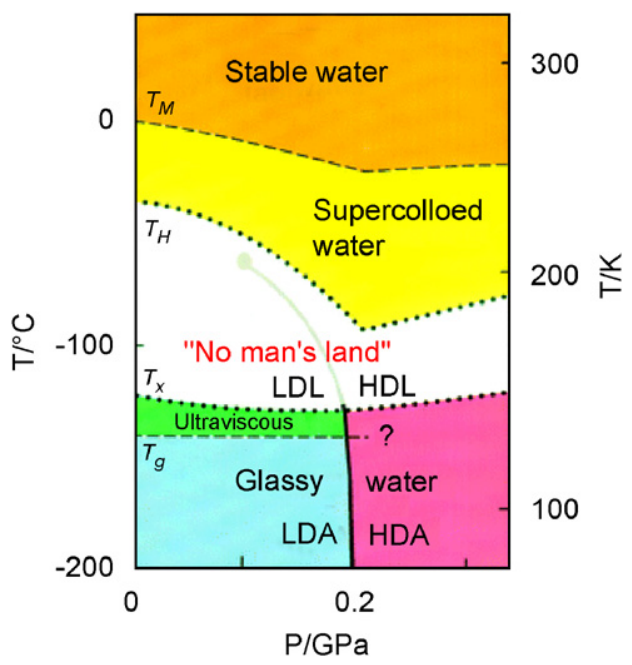

Fig. 4. Schematic illustration indicating the various phases of liquid water (color-coded). This figure is courtesy of Dr. O. Mishima. 
subject to the charge "garbage-in, garbage-out"-you get out what you put in. All computer models of complex systems such as liquid water are of necessity simplifications.

Current experiments on this problem are of two sorts. The first is a set of experiments inspired by Mishima that involves probing the No Man's Land by studying the metastable extensions of the melting lines of the various high-pressure polymorphs of ice: ice III, ice V, ice IV, and ice XII [13,14]. Two of these lines clearly display "kinks." Since the slope of any melting line is the difference of the volume change divided by the entropy change of the two phases that coexist at that line, if there is a change in slope there must be a change in these quantities. Since there is no change in the crystal part, there must be a change in the liquid part. This means the liquid must undergo a jump in either its volume or its entropy or both. That is the definition of a first-order phase transition. A second set of experiments is being carried out in the MIT group of Chen and the Messina group of Mallamace, which have stimulated much of our recent work.

\section{Recent work}

Next we describe, for two water models displaying a liquid-liquid critical point, the relation between changes in dynamic and thermodynamic anomalies arising from the presence of the liquid-liquid critical point. We find a correlation between the dynamic fragility transition and the locus of specific heat maxima $C_{P}^{\max }$ ("Widom line") emanating from the critical point. Our findings are consistent with a possible relation between the previously hypothesized liquid-liquid phase transition and the transition in the dynamics recently observed in neutron scattering experiments on confined water. More generally, we argue that this connection between $C_{P}^{\max }$ and dynamic crossover is not limited to the case of water, a hydrogen bonded network liquid, but is a more general feature of crossing the Widom line. Specifically, we also study the Jagla potential, a spherically symmetric two-scale potential known to possess a liquid-liquid critical point, in which the competition between two liquid structures is generated by repulsive and attractive ramp interactions. Using molecular dynamics (MD) simulations, we also investigate the relation between the dynamic transitions of biomolecules (lysozyme and DNA) and the dynamic and thermodynamic properties of hydration water. We find that the dynamic transition of the macromolecules, sometimes called a "protein glass transition", occurs at the temperature of dynamic crossover in the diffusivity of hydration water, and also coincides with the maxima of the isobaric specific heat $C_{P}$ and the temperature derivative of the orientational-order parameter. We relate these findings to the hypothesis of a liquid-liquid critical point in water. Our simulations are consistent with the possibility that the protein glass transition results from a change in the behavior of hydration water, specifically from crossing the Widom line.

\subsection{The Widom line}

By definition, in a first-order phase transitions, thermodynamic state functions such as density $\rho$ and enthalpy $H$ discontinuously change as we cool the system along a path crossing the equilibrium coexistence line [Fig. 5(a), path $\beta$ ]. In a real experiment, this discontinuous change may not occur at the coexistence line since a substance can remain in a supercooled metastable phase until a limit of stability (a spinodal) is reached [15] [Fig. 5(b), path $\beta$ ]. If the system is cooled isobarically along a path above the liquid-gas critical pressure $P_{c}$ [Fig. 5(b), path $\alpha$ ], the state functions continuously change from the values characteristic of a high temperature phase (gas) to those characteristic of a low temperature phase (liquid). The thermodynamic response functions which are the derivatives of the state functions with respect to temperature [e.g., isobaric heat capacity $\left.C_{P} \equiv(\partial H / \partial T)_{P}\right]$ have maxima at temperatures denoted by $T_{\max }(P)$. Remarkably, these maxima are still prominent far above the critical pressure [17-21], and the values of the response functions at $T_{\max }(P)$ (e.g., $C_{P}^{\max }$ ) diverge as the critical point is approached. The lines of the maxima for different response functions asymptotically approach one another as the critical point is approached, since all response functions become expressible in terms of the correlation length. This asymptotic line is sometimes called the Widom line, and is often regarded as an extension of the coexistence line into the "one-phase regime."

Water's anomalies have been hypothesized to be related to the existence of a line of a first-order liquid-liquid phase transition terminating at a liquid-liquid critical point $[10,13,15,16]$, located below 

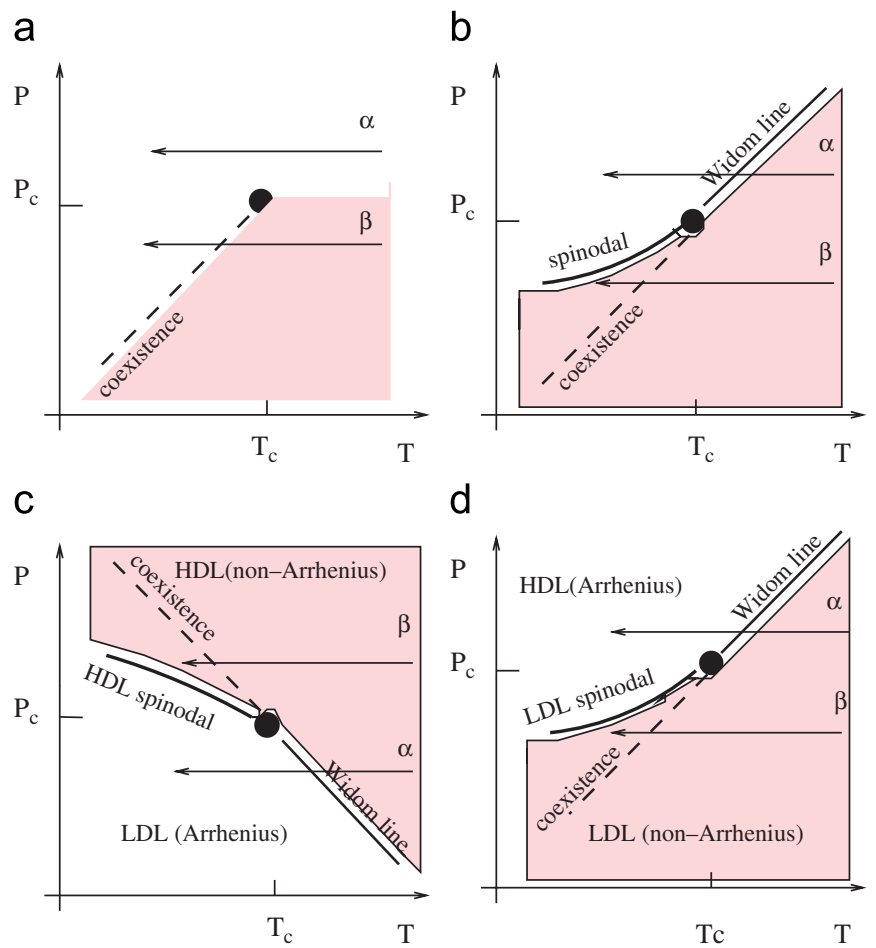

Fig. 5. (a) Schematic phase diagram for the critical region associated with a liquid-gas critical point. Shown are the two features displaying mathematical singularities, the critical point (closed circles) and the liquid-gas coexistence (bold dashed curve). (b) Same as (a) with the addition of the gas-liquid spinodal and the Widom line. Along the Widom line, thermodynamic response functions have extrema in their $T$ dependence. The path $\alpha$ denotes a path along which the Widom line is crossed. Path $\beta$ denotes a path meeting the coexistence line. (c) A hypothetical phase diagram for water of possible relevance to the recent neutron scattering experiments by Chen et al. [39,40] on confined water. The liquid-liquid coexistence, which has a negative sloped coexistence line, generates a Widom line which extends below the critical point, suggesting that water may exhibit a dynamic crossover (non-Arrhenius-to-Arrhenius) transition for $P<P_{c}$ (path $\alpha$ ), while no dynamic changes will occur above the critical point (path $\beta$ ). (d) A sketch of the $P-T$ phase diagram for the two-scale Jagla model. For the Jagla potential, as well as for the double-step potential [41], the liquid-liquid phase transition line has a positive slope. Upon cooling at constant pressure above the critical point (path $\alpha$ ), the liquid changes from a low density state (characterized by a non-glassy Arrhenius dynamics) to a high density state (characterized by glassy Arrhenius dynamics with much larger activation energy) as the path crosses the Widom line. Upon cooling at constant pressure below the critical point (path $\beta$ ), the liquid remains in the LDL phase as long as path $\beta$ does not cross the LDL spinodal line. Thus one does not expect any change in the dynamic behavior along the path $\beta$, except upon approaching to glass transition where one can expect the non-Arrhenius behavior characterized by the Vogel-Fulcher-Tamman (VFT) fit.

the homogeneous nucleation line in the deep supercooled region of the phase diagram - sometimes called the "no-man's land" because it is difficult to make direct measurements on the bulk liquid phase [13]. In supercooled water, the liquid-liquid coexistence line and the Widom line have negative slopes. Thus, if the system is cooled at constant pressure $P_{0}$, computer simulations suggest that for $P_{0}<P_{c}$ [Fig. 5(c), path $\alpha$ ] experimentally measured quantities will change dramatically but continuously in the vicinity of the Widom line (with huge fluctuations as measured by, e.g., $C_{P}$ ) from those resembling the HDL to those resembling the LDL. For $P_{0}>P_{c}$ [Fig. 5(d), path $\beta$ ], experimentally measured quantities will change discontinuously if the coexistence line is actually seen. However, the coexistence line can be difficult to detect in a pure system due to metastability, and changes will occur only when the spinodal is approached where the HDL phase is no longer stable. The changes in behavior may include not only static quantities like response functions [17-21] but also dynamic quantities like diffusivity.

In the case of water, a significant change in dynamical properties has been suggested to take place in deeply supercooled states [16,22-25]. Unlike other network forming materials [26], water behaves as a non-Arrhenius liquid in the experimentally accessible window $[16,27,28]$. Based on analogies with other network forming 
liquids and with the thermodynamic properties of the amorphous forms of water, it has been suggested that, at ambient pressure, liquid water should show a dynamic crossover from non-Arrhenius behavior at high $T$ to Arrhenius behavior at low $T$ [24,29-33]. Using Adam-Gibbs theory [34], the dynamic crossover in water was related to the $C_{P}^{\max }$ line $[22,35]$. Also, a dynamic crossover has been associated with the liquid-liquid phase transition in simulations of silicon and silica [36,37]. Recently a dynamic crossover in confined water was studied experimentally [38-40,42] since nucleation can be avoided in confined geometries. In this work, we interpret recent experiments on water [39,40,42] as arising from the presence of the hypothesized liquid-liquid critical point, which gives rise to a Widom line and an associated fragility transition [Fig. 5(c), path $\alpha$.

\subsection{Results for bulk water}

Using MD simulations [43], $\mathrm{Xu}$ et al. [44] studied three models, each of which has a liquid-liquid critical point. Two of the models (the TIP5P [45] and the ST2 [46]) treat water as a multiple site rigid body, interacting via electrostatic site-site interactions complemented by a Lennard-Jones potential. The third model is the spherical "two-scale" Jagla potential with attractive and repulsive ramps which has been studied in the context of liquid-liquid phase transitions and liquid anomalies [21,30-32,47,48]. For all three models, Xu et al. evaluated the loci of maxima of the relevant response functions, compressibility and specific heat, which coincide close to the critical point and give rise to the Widom line. They found evidence that, for all three potentials, the dynamic crossover occurs just when the Widom line is crossed (Fig. 6).

These findings are consistent with the possibility that the observed dynamic crossover along path $\alpha$ is related to the behavior of $C_{P}$, suggesting that enthalpy or entropy fluctuations may have a strong influence on the dynamic properties [21,22,37]. Indeed, as the thermodynamic properties change from the high-temperature side of the Widom line to the low-temperature side, $(\partial S / \partial T)_{P}=C_{P} / T>0$ implies that the entropy must decrease. The entropy decrease is most pronounced at the Widom line when $C_{P}=C_{P}^{\max }$. Since the configurational part of the entropy, $S_{\text {conf }}$, makes the major contribution to $S$, we expect that $S_{\text {conf }}$ also decreases sharply on crossing the Widom line.

According to Adam-Gibbs theory [34], $D \sim \exp \left(-A / T S_{\text {conf }}\right)$. Hence, we expect that $D$ sharply decreases upon cooling at the Widom line. If $S_{\text {conf }}$ does not change appreciably with $T$, then the Adam-Gibbs equation predicts an Arrhenius behavior of $D$. For both water and the Jagla model, crossing the Widom line is associated with the change in the behavior of the diffusivity. (i) In the case of water, $D$ changes from nonArrhenius to Arrhenius behavior, while the structural and thermodynamic properties change from those resembling HDL to those resembling LDL, due to the negative slope of the Widom line. (ii) For the Jagla potential, $D$ changes from Arrhenius to non-Arrhenius while the structural and thermodynamic properties change from those resembling LDL to those resembling HDL, due to the positive slope of the Widom line.

Thus these results for bulk water are consistent with the experimental observation in confined water of: (i) a fragility transition for $P<P_{c}$ [39,40], and (ii) a peak in $C_{P}$ upon cooling water at atmospheric pressure [49], so this work offers a plausible interpretation of the results of Ref. [40] as supporting the existence of a hypothesized liquid-liquid critical point.

Fig. 6. (a) Relevant part of the phase diagram for the TIP5P potential, showing the liquid-liquid critical point $C$ at $P_{c}=320 \mathrm{MPa}$ and $T_{c}=217 \mathrm{~K}$, the line of maximum of isobaric specific heat $C_{P}^{\max }$ and the line of maximum of isothermal compressibility $K_{T}^{\max }$. (b) $D$ as a function of $T$ for $P=100 \mathrm{MPa}$ (path $\alpha$ ). At high temperatures, $D$ behaves like that of a non-Arrhenius liquid and can be fit by $D \sim\left(T-T_{M C T}\right)^{\gamma}$ (also shown in the inset) where $T_{M C T}=220 \mathrm{~K}$ and $\gamma=1.942$, while at low temperatures the dynamic behavior changes to that of a liquid where $D$ is Arrhenius. (c) The same for the ST2 potential. The liquid-liquid critical point $C$ is located at $P_{c}=246 \mathrm{MPa}$ and $T_{c}=146 \mathrm{~K}$. (d) $D$ as a function of $T$ for $P=100 \mathrm{MPa}$ (path $\alpha$ ). At high temperatures, $D$ behaves like that of a non-Arrhenius liquid and can be fit by $D \sim\left(T-T_{M C T}\right)^{\gamma}$ (also shown in the inset) where $T_{M C T}=239 \mathrm{~K}$ and $\gamma=1.57$, while at low temperatures the dynamic behavior changes to that of a liquid where $D$ is Arrhenius. (e) Phase diagram for the Jagla potential in the vicinity of the liquid-liquid phase transition. Shown are the liquid-liquid critical point $C$ located at $P_{c}=0.24$ and $T_{c}=0.37$, the line of isobaric specific heat maximum $C_{P}^{\max }$, the line of isothermal compressibility $K_{T}^{\max }$, and spinodal lines. (f) $D$ as a function of $T$ for $P=0.250$ (squares, path $\alpha$ ) and $P=0.225$ (triangles, path $\beta$ ). Along path $\alpha$, one can see a sharp crossover from the high temperature Arrhenius behavior $D \approx$ $\exp (-1.53 / T)$ with lower activation energy to a low temperature Arrhenius behavior $D \approx \exp (-6.3 / T)$ with high activation energy, which is a characteristic of the HDL. Along path $\beta$, there is no sharp changes near the critical point, because the liquid remains in the LDL phase. However, near the glass transition, LDL exhibits a non-Arrhenius behavior characterized by the VFT fit at very low temperature. 


\subsection{Glass transition in biomolecules}

Next we explore the hypothesis [50] that the observed glass transition in biomolecules [12,51-63,71] is related to the liquid-liquid phase transition using MD simulations. Specifically, Kumar et al. [50] studied the dynamic and thermodynamic behavior of lysozyme and DNA in hydration TIP5P water, by means of the software package GROMACS [64] for: (i) an orthorhombic form of hen egg-white lysozyme [65] and

a

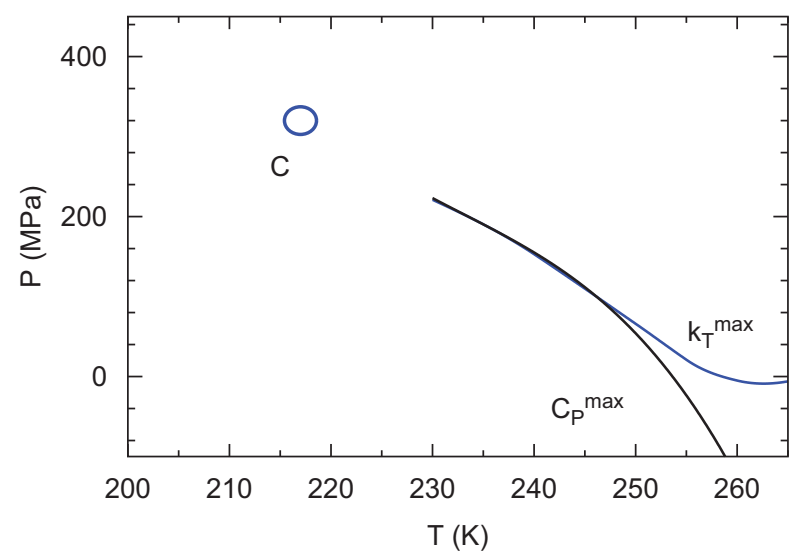

C

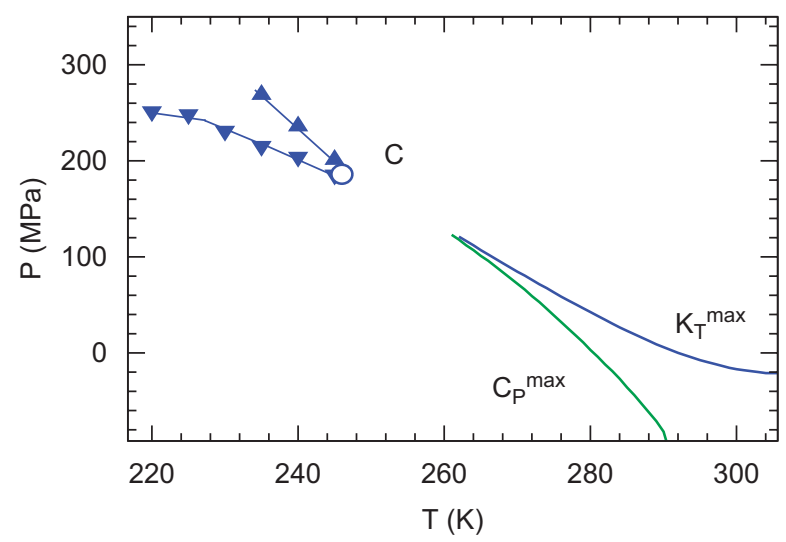

e

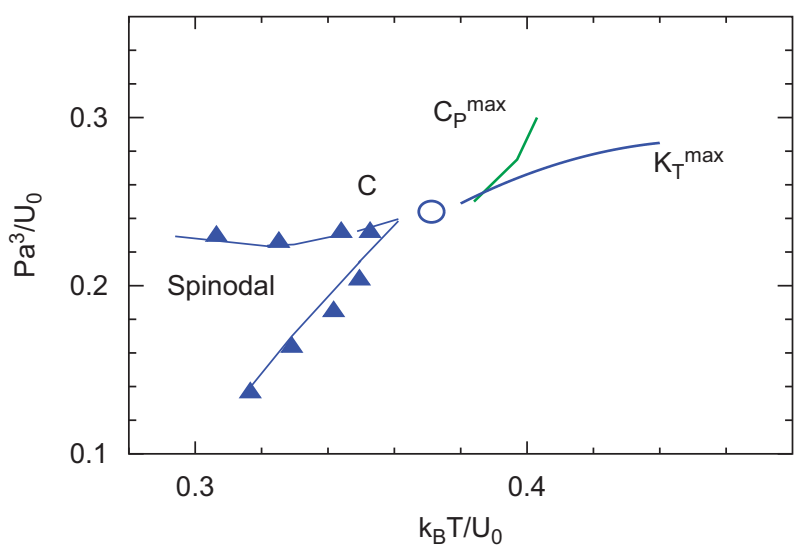

b

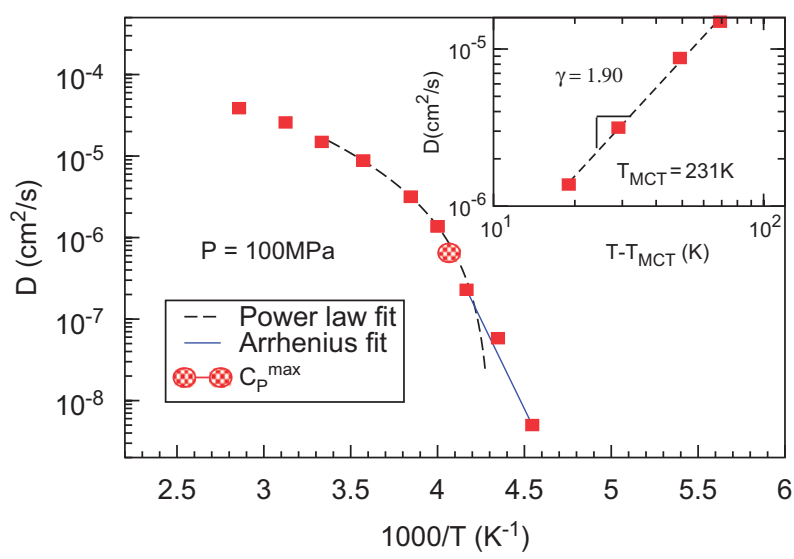

d

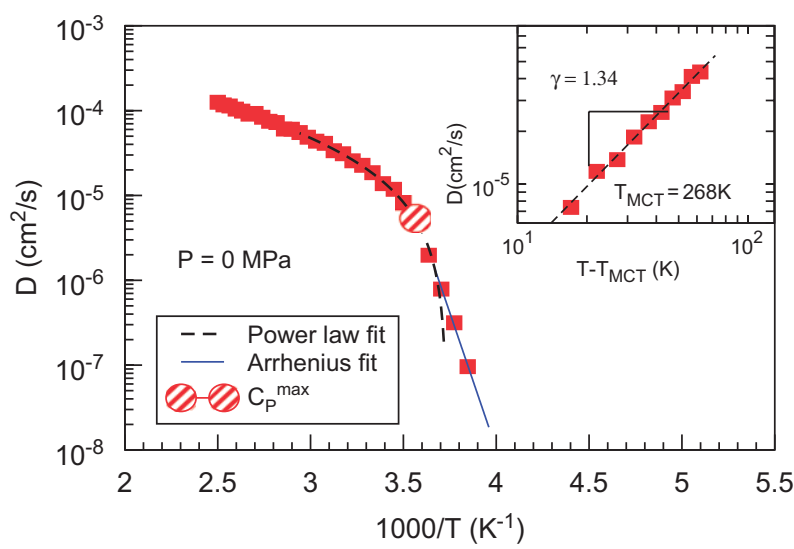

f

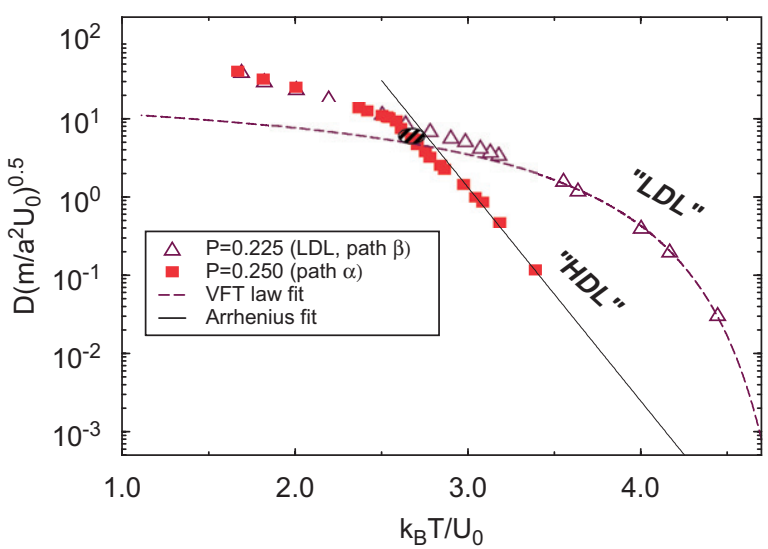


a

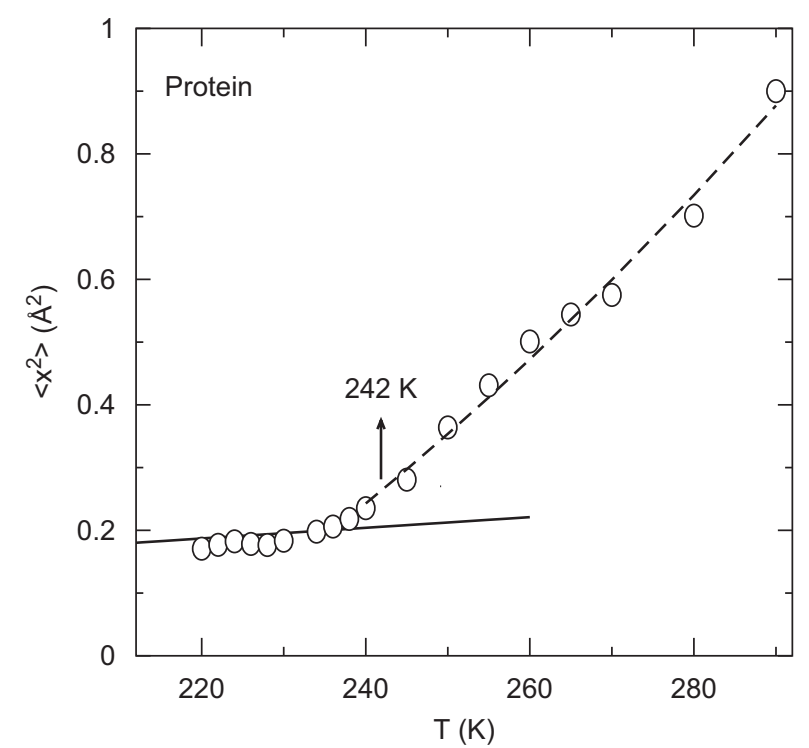

b

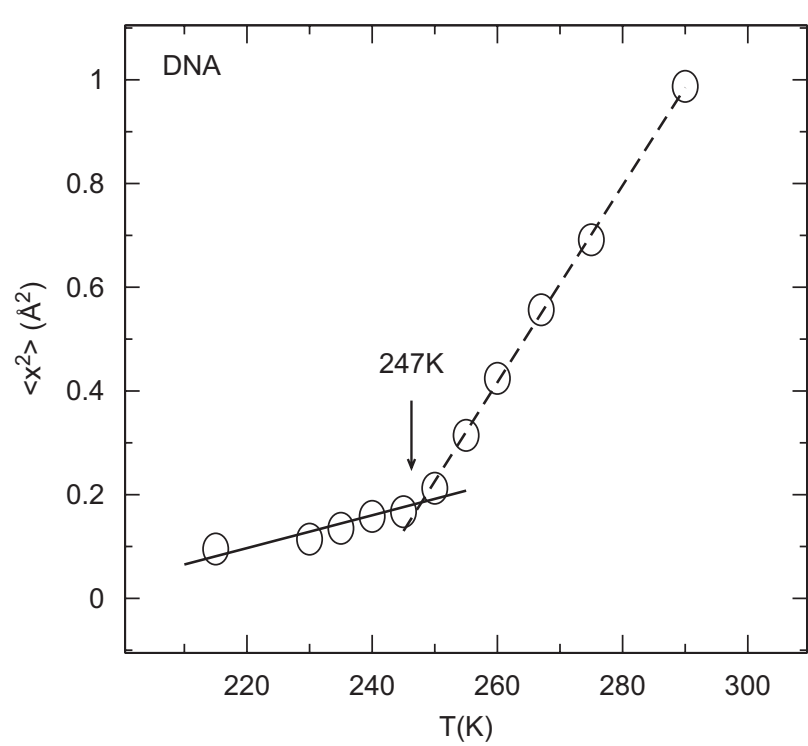

Fig. 7. Mean square fluctuation of: (a) lysozyme, and (b) DNA showing that there is a transition around $T_{p} \approx 242 \pm 10 \mathrm{~K}$ for lysozyme and around $T_{p} \approx 247 \pm 10 \mathrm{~K}$ for DNA. For very low $T$ one would expect a linear increase of $\left\langle x^{2}\right\rangle$ with $T$, as a consequence of harmonic approximation for the motion of residues. At high $T$, the motion becomes non-harmonic and we fit the data by a polynomial. We determine the dynamic crossover temperature $T_{p}$ from the crossing of the linear fit for low $T$ and the polynomial fit for high $T$. We determine the error bars by changing the number of data points in the two fitting ranges.

(ii) a Dickerson dodecamer DNA [66] at constant pressure $P=1 \mathrm{~atm}$, several constant temperatures $T$, and constant number of water molecules $N$ (NPT ensemble).

The simulation results for the mean square fluctuations $\left\langle x^{2}\right\rangle$ of both protein and DNA are shown in Fig. 7(a). Kumar et al. calculated the mean square fluctuations $\left\langle x^{2}\right\rangle$ of the biomolecules from the equilibrated configurations, first for each atom over $1 \mathrm{~ns}$, and then averaged over the total number of atoms in the biomolecule. They find that $\left\langle x^{2}\right\rangle$ changes its functional form below $T_{p} \approx 245 \mathrm{~K}$, for both lysozyme [Fig. 7(a)] and DNA [Fig. 7(b)].

Kumar et al. next calculated $C_{P}$ by numerical differentiation of the total enthalpy of the system (protein and water) by fitting the simulation data for enthalpy with a fifth-order polynomial, and then taking the derivative with respect to $T$. Fig. 4(a) and (b) displays maxima of $C_{P}(T)$ at $T_{W} \approx 250 \pm 10 \mathrm{~K}$ for both biomolecules.

Further, to describe the quantitative changes in structure of hydration water, Kumar et al. calculated the local tetrahedral-order parameter $Q$ [67-70] for hydration water surrounding lysozyme and DNA. Fig. 4(c) and (d) shows that the rate of increase of $Q$ has a maximum at $245 \pm 10 \mathrm{~K}$ for lysozyme and DNA hydration water respectively; the same temperatures of the crossover in the behavior of mean square fluctuations.

Upon cooling, the diffusivity of hydration water exhibits a dynamic crossover from non-Arrhenius to Arrhenius behavior at the crossover temperature $T_{\times} \approx 245 \pm 10 \mathrm{~K}$ [Fig. 8(e)]. The coincidence of $T_{\times}$with $T_{p}$ within the error bars indicates that the behavior of the protein is strongly coupled with the behavior of the surrounding solvent, in agreement with recent experiments [51]. Note that $T_{\times}$is much higher than the glass transition temperature, estimated for TIP5P as $T_{g}=215 \mathrm{~K}$ [72]. Thus this crossover is not likely to be related to the glass transition in water.

The fact that $T_{p} \approx T_{\times} \approx T_{W}$ is evidence of the correlation between the changes in protein fluctuations [Fig. 7(a)] and the hydration water thermodynamics [Fig. 8(a)]. Thus these results are consistent with the possibility that the protein glass transition is related to the Widom line (and hence to the hypothesized liquid-liquid critical point). Crossing the Widom line corresponds to a continuous but rapid transition of the properties of water from those resembling the properties of a local HDL structure for $T>T_{W}(P)$ to those resembling the properties of a local LDL structure for $T<T_{W}(P)[40,44]$. A consequence is the expectation 

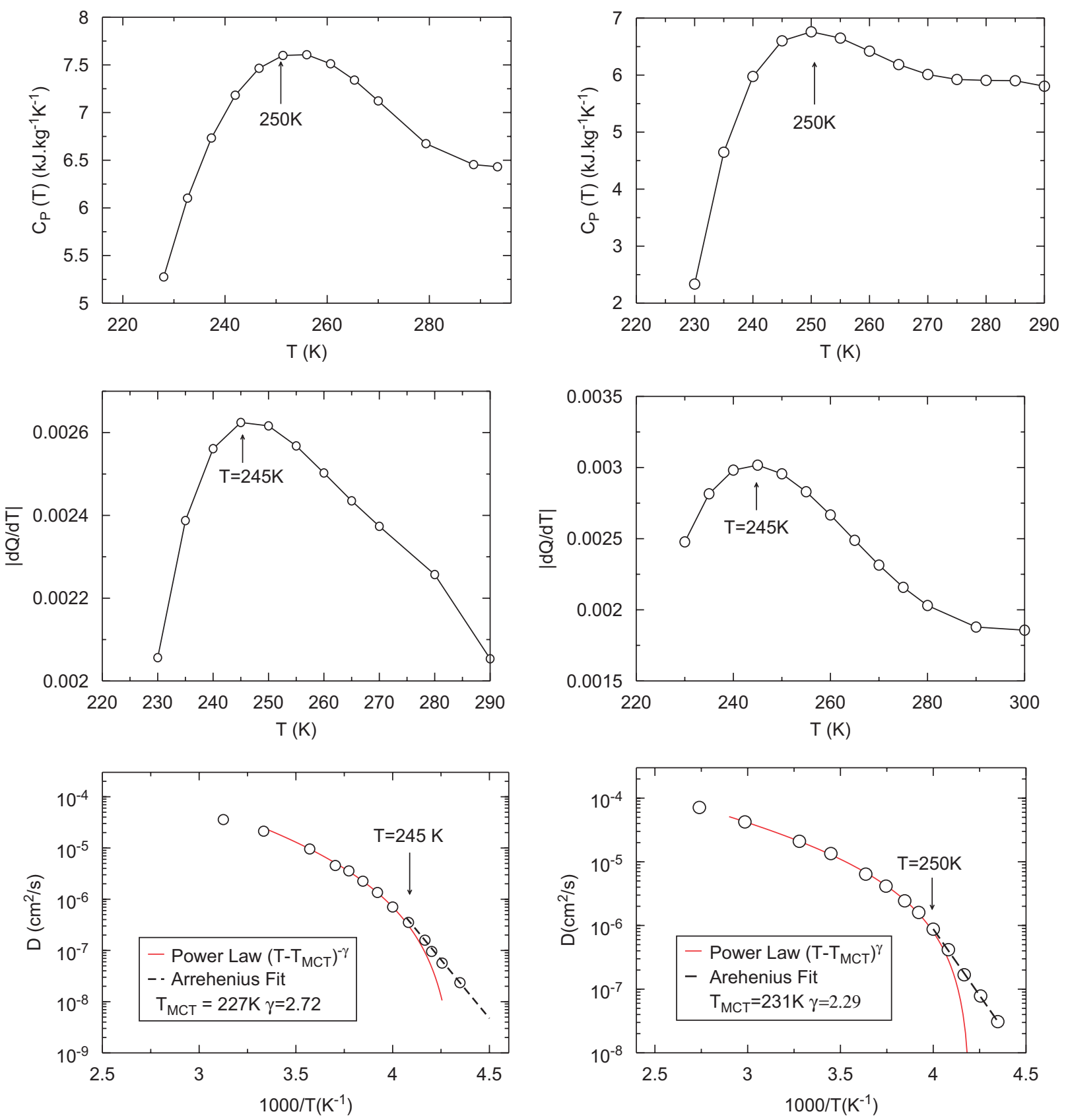

Fig. 8. The specific heat of the combined system: (a) lysozyme and water, and (b) DNA and water, display maxima at $250 \pm 10$ and $250 \pm 12 \mathrm{~K}$, respectively, which are coincident within the error bars with the temperature $T_{p}$ where the crossover in the behavior of $\left\langle x^{2}\right\rangle$ is observed in Fig. 5. Derivative with respect to temperature of the local tetrahedral order parameter $Q$ for: (c) lysozyme and (d) DNA hydration water. A maximum in $|\mathrm{d} Q / \mathrm{d} T|$ at Widom line temperature suggests that the rate of change of local tetrahedrality of hydration water has a maximum at the Widom line. Diffusion constant of hydration water surrounding: (e) lysozyme, and (f) DNA shows a dynamic transition from a power law behavior to an Arrhenius behavior at $T_{\times} \approx 245 \pm 10 \mathrm{~K}$ for lysozyme and $T_{\times} \approx 250 \pm 10 \mathrm{~K}$ for DNA, around the same temperatures where the behavior of $\left\langle x^{2}\right\rangle$ has a crossover, and $C_{P}$ and $|\mathrm{d} Q / \mathrm{d} T|$ have maxima.

that the fluctuations of the protein residues in predominantly LDL-like water (more ordered and more rigid) just below the Widom line should be smaller than the fluctuations in predominantly HDL-like water (less ordered and less rigid) just above the Widom line. 
The quantitative agreement of the results for both DNA and lysozyme [Figs. 3 and 4] suggests that it is indeed the changes in the properties of hydration water that are responsible for the changes in dynamics of the protein and DNA biomolecules. Our results are in qualitative agreement with recent experiments on hydrated protein and DNA [73] which found the crossover in side-chain fluctuations at $T_{p} \approx 225 \mathrm{~K}$.

\section{Outlook}

It is possible that other phenomena that appear to occur on crossing the Widom line are in fact not coincidences, but are related to the changes in local structure that occur when the system changes from the "HDL-like" side to the "LDL-like" side. In this work we concentrated on reviewing the evidence for changes in dynamic transport properties, such as diffusion constant and relaxation time. Additional examples include: (1) a breakdown of the Stokes-Einstein relation for $T<T_{W}(P)$ [74-79], (2) systematic changes in the static structure factor $S(q)$ and the corresponding pair correlation function $g(r)$ revealing that for $T<T_{W}(P)$ the system more resembles the structure of LDL than HDL, (3) appearance for $T<T_{W}(P)$ of a shoulder in the dynamic structure factor $S(q, \omega)$ at a frequency $\omega \approx 60 \mathrm{~cm}^{-1} \approx 2 \mathrm{THz}$ [51], (4) rapid increase in hydrogen bonding degree for $T<T_{W}(P)$ [80,81], (5) a minimum in the density at low temperature [82], and (6) a scaled equation of state near the critical point [83]. It is important to know how general a given phenomenon is, such as crossing the Widom line which by definition is present whenever there is a critical point. Using data on other liquids which have local tetrahedral symmetry, such as silicon and silica, which appear to also display a liquid-liquid critical point and hence must possess a Widom line emanating from this point into the one-phase region. For example, after this work was completed, we learned of interesting new work on silicon, which also interprets structural changes as arising from crossing the Widom line of silicon [84]. It might be interesting to test the effect of the Widom line on simple model systems that display a liquid-liquid critical point, such as two-scale symmetric potentials of the sort recently studied by Xu and collaborators [21].

\section{Acknowledgments}

This work, supported by the NSF Chemistry Division, has been carried out with many collaborators and has been heavily influenced by a number of experimentalists including S.-H. Chen $[44,50,77,82]$, L. Liu [39,40], F. Mallamace [85,86], O. Mishima [13,87,88], J. Teixeira [89-91], M.-C. Bellissent [92-95], and H. Reichert [96].

\section{Note Added in Proof}

After this work was completed, Mallamace and his collaborators succeeded in locating the Widom line by finding a clearcut maximum in the coefficient of thermal expansion, at $T_{W} \approx 225 \mathrm{~K}$ [97], which remarkably is the same temperature as the specific heat maximum [49].

Also, private discussions with Jacob Klein reveal a possible reason for why confined water does not freeze at $-38^{\circ} \mathrm{C}$, the bulk homogeneous nucleation temperature: Klein and co-workers [98] noted that confined water behaves differently than typical liquids in that it water does not experience the huge increase in viscosity characteristic of other strongly confined liquids. They interpret this experimental finding as arising from the fact that strong confinement hampers the formation of a hydrogen bonded network, and we know from classic work of Linus Pauling that without the extensive hydrogen bonded network, water's freezing temperature will be depressed by more than $100^{\circ}$. Thus confinement reduces the extent of the hydrogen bonded network and hence lowers the freezing temperature, but leaves the key tetrahedral local geometry unchanged.

\section{References}

[1] T. Andrews, Philos. Trans. 159 (1869) 575.

[2] V. Brazhkin, S.V. Buldyrev, V.N. Ryzhov, H.E. Stanley (Eds.), New Kinds of Phase Transitions: Transformations in Disordered Substances, Proceedings of the NATO Advanced Research Workshop, Volga River, Kluwer, Dordrecht, 2002. 
[3] A. Geiger, H.E. Stanley, Low-density patches in the hydrogen-bonded network of liquid water: evidence from molecular dynamics computer simulations, Phys. Rev. Lett. 49 (1982) 1749-1752.

[4] M. Canpolat, F.W. Starr, M.R. Sadr-Lahijany, A. Scala, O. Mishima, S. Havlin, H.E. Stanley, Local structural heterogeneities in liquid water under pressure, Chem. Phys. Lett. 294 (1998) 9-12.

[5] F. Sciortino, P. Poole, H.E. Stanley, S. Havlin, Lifetime of the hydrogen bond network and gel-like anomalies in supercooled water, Phys. Rev. Lett. 64 (1990) 1686-1689.

[6] A. Geiger, H.E. Stanley, Tests of universality of percolation exponents for a 3-dimensional continuum system of interacting waterlike particles, Phys. Rev. Lett. 49 (1982) 1895-1898.

[7] P.C. Hemmer, G. Stell, Phys. Rev. Lett. 24 (1970) 1284.

[8] M.R. Sadr-Lahijany, A. Scala, S.V. Buldyrev, H.E. Stanley, Phys. Rev. Lett. 81 (1998) 4895.

[9] A. Scala, M.R. Sadr-Lahijany, N. Giovambattista, S.V. Buldyrev, H.E. Stanley, Phys. Rev. E 63 (2001) 041202.

[10] P.H. Poole, F. Sciortino, U. Essmann, H.E. Stanley, Phase behavior of metastable water, Nature 360 (1992) $324-328$.

[11] O. Mishima, J. Chem. Phys. 100 (1994) 5910.

[12] M. Yamada, S. Mossa, H.E. Stanley, F. Sciortino, Phys. Rev. Lett. 88 (2002) 195701.

[13] O. Mishima, H.E. Stanley, Decompression-induced melting of ice IV and the liquid-liquid transition in water, Nature 392 (1998) 164-168.

[14] O. Mishima, Phys. Rev. Lett. 85 (2002) 334.

[15] P.G. Debenedetti, H.E. Stanley, The physics of supercooled and glassy water, Phys. Today 56 (issue 6) (2003) $40-46$.

[16] P.G. Debenedetti, J. Phys. Condens. Matter 15 (2003) R1669-R1726.

[17] M.A. Anisimov, J.V. Sengers, J.M.H. Levelt-Sengers, in: D.A. Palmer, R. Fernandez-Prini, A.H. Harvey (Eds.), Aqueous System at Elevated Temperatures and Pressures: Physical Chemistry in Water, Steam and Hydrothermal Solutions, Elsevier, Amsterdam, 2004, pp. 29-71.

[18] J.M.H. Levelt, Measurements of the compressibility of argon in the gaseous and liquid phase, Ph.D. Thesis, University of Amsterdam, Van Gorkum and Co., Assen, 1958.

[19] A. Michels, J.M.H. Levelt, G. Wolkers, Physica 24 (1958) 769-794.

[20] A. Michels, J.M.H. Levelt, W. de Graaff, Physica 24 (1958) 659-671.

[21] L. Xu, S.V. Buldyrev, C.A. Angell, H.E. Stanley, Thermodynamics and dynamics of the two-scale spherically symmetric Jagla ramp model of anomalous liquids, Phys. Rev. E 74 (2006) 031108.

[22] F.W. Starr, C.A. Angell, H.E. Stanley, Prediction of entropy and dynamic properties of water below the homogeneous nucleation temperature, Physica A 323 (2003) 51-66.

[23] C.A. Angell, Ann. Rev. Phys. Chem. 55 (2004) 559-583.

[24] C.A. Angell, J. Phys. Chem. 97 (1993) 6339-6341.

[25] P. Kumar, S.V. Buldyrev, F. Starr, N. Giovambattista, H.E. Stanley, Thermodynamics, structure, and dynamics of water confined between hydrophobic plates, Phys. Rev. E 72 (2005) 051503.

[26] J. Horbach, W. Kob, Phys. Rev. B 60 (1999) 3169-3181.

[27] E.W. Lang, H.D. Lüdemann, Angew. Chem. Intl. Ed. Engl. 21 (2004) 315-329.

[28] F.X. Prielmeier, E.W. Lang, R.J. Speedy, H.D. Lüdemann, Phys. Rev. Lett. 59 (1987) 1128-1131.

[29] K. Ito, C.T. Moynihan, C.A. Angell, Nature 398 (1999) 492-495.

[30] E.A. Jagla, J. Chem. Phys. 111 (1999) 8980-8986.

[31] E.A. Jagla, J. Phys. Condens. Matter 11 (1999) 10251-10258.

[32] E.A. Jagla, Phys. Rev. E 63 (2001) 061509.

[33] H. Tanaka, J. Phys. Condens. Matter 15 (2003) L703-L711.

[34] G. Adam, J.H. Gibbs, J. Chem. Phys. 43 (1965) 139-146.

[35] P.H. Poole, I. Saika-Voivod, F. Sciortino, J. Phys. Condens. Matter 17 (2005) L431-L437.

[36] S. Sastry, C.A. Angell, Nat. Mater. 2 (2003) 739-743.

[37] I. Saika-Voivod, P.H. Poole, F. Sciortino, Nature 412 (2001) 514-517.

[38] R. Bergman, J. Swenson, Nature 403 (2000) 283-286.

[39] A. Faraone, L. Liu, C.-Y. Mou, C.-W. Yen, S.-H. Chen, J. Chem. Phys. 121 (2004) 10843-10846.

[40] L. Liu, S.-H. Chen, A. Faraone, C.-W. Yen, C.-Y. Mou, Phys. Rev. Lett. 95 (2005) 117802.

[41] G. Franzese, G. Malescio, A. Skibinsky, S.V. Buldyrev, H.E. Stanley, Generic mechanism for generating a liquid-liquid phase transition, Nature 409 (2001) 692-695 (cond-mat/0102029).

[42] F.M. Mallamace, M. Broccio, C. Corsaro, A. Faraone, U. Wanderlingh, L. Liu, C.-Y. Mou, S.-H. Chen, J. Chem. Phys. 124 (2006) 161102.

[43] D.C. Rapaport, The Art of Molecular Dynamics Simulation, Cambridge University Press, Cambridge, 1995.

[44] L. Xu, P. Kumar, S.V. Buldyrev, S.-H. Chen, P.H. Poole, F. Sciortino, H.E. Stanley, Relation between the Widom line and the dynamic crossover in systems with a liquid-liquid critical point, Proc. Natl. Acad. Sci. 102 (2005) 16558-16562.

[45] M.W. Mahoney, W.L. Jorgensen, J. Chem. Phys. 112 (2000) 8910-8922.

[46] F.H. Stillinger, A. Rahman, J. Chem. Phys. 57 (1972) 1281-1292.

[47] P. Kumar, S.V. Buldyrev, F. Sciortino, E. Zaccarelli, H.E. Stanley, Static and dynamic anomalies in a repulsive spherical ramp liquid: theory and simulation, Phys. Rev. E 72 (2005) 021501.

[48] L. Xu, I. Ehrenberg, S.V. Buldyrev, H.E. Stanley, Relationship between the liquid-liquid phase transition and dynamic behavior in the Jagla model, J. Phys. Condens. Matter 18 (2006) S2239-S2246. 
[49] S. Maruyama, K. Wakabayashi, M. Oguni, AIP Conf. Proc. 708 (2004) 675-676.

[50] P. Kumar, Z. Yan, L. Xu, M.G. Mazza, S.V. Buldyrev, S.-H. Chen, S. Sastry, H.E. Stanley, Glass transition in biomolecules and the liquid-liquid critical point of water, Phys. Rev. Lett. 97 (2006) 177802.

[51] S.-H. Chen, L. Liu, E. Fratini, P. Baglioni, A. Faraone, E. Mamontov, The observation of fragile-to-strong dynamic crossover in protein hydration water, Proc. Natl. Acad. Sci. USA 103 (2006) 9012.

[52] J.M. Zanotti, M.-C. Bellissent-Funel, J. Parrello, Biophys. J. 76 (1999) 2390.

[53] D. Ringe, G.A. Petsko, Biophys. Chem. 105 (2003) 667.

[54] J. Wang, P. Cieplak, P.A. Kollman, J. Compd. Chem. 21 (2000) 1049; E.J. Sorin, V.S. Pande, Biophys. J. 88 (2005) 2472.

[55] B.F. Rasmussen, M. Ringe, G.A. Petsko, Nature 357 (1992) 423.

[56] D. Vitkup, D. Ringe, G.A. Petsko, M. Karplus, Nat. Struct. Biol. 7 (2000) 34.

[57] A.P. Sokolov, H. Grimm, A. Kisliuk, A.J. Dianoux, J. Chem. Phys. 110 (1999) 7053.

[58] W. Doster, S. Cusack, W. Petry, Nature 338 (1989) 754.

[59] J. Norberg, L. Nilsson, Proc. Natl. Acad. Sci. USA 93 (1996) 10173.

[60] M. Tarek, D.J. Tobias, Phys. Rev. Lett. 88 (2002) 138101; M. Tarek, D.J. Tobias, Biophys. J. 79 (2000) 3244.

[61] H. Hartmann, F. Parak, W. Steigemann, G.A. Petsko, D.R. Ponzi, H. Frauenfelder, Proc. Natl. Acad. Sci. USA 79 (1982) 4067.

[62] A.L. Tournier, J. Xu, J.C. Smith, Biophys. J. 85 (2003) 1871.

[63] A.L. Lee, A.J. Wand, Nature 411 (2001) 501.

[64] E. Lindahl, B. Hess, D. van der Spoel, J. Mol. Modeling 7 (2001) 306.

[65] P.J. Artymiuk, C.C.F. Blake, D.W. Rice, K.S. Wilson, Acta Crystallogr. B 38 (1982) 778.

[66] H.R. Drew, R.M. Wing, T. Takano, C. Broka, S. Tanaka, K. Itakura, R.E. Dickerson, Proc. Natl. Acad. Sci. USA 78 (1981) 2179.

[67] J.R. Errington, P.G. Debenedetti, Nature 409 (2001) 318.

[68] Z. Yan, S.V. Buldyrev, N. Giovambattista, H.E. Stanley, Structural order for one-scale and two-scale potentials, Phys. Rev. Lett. 95 (2005) 130604.

[69] Z. Yan, S.V. Buldyrev, N. Giovambattista, P.G. Debenedetti, H.E. Stanley, Family of tunable spherically-symmetric potentials that span the range from hard spheres to water-like behavior, Phys. Rev. E 73 (2006) 051204.

[70] Z. Yan, S.V. Buldyrev, P. Kumar, N. Giovambattista, P.G. Debenedetti, H.E. Stanley, Structure of the first- and second- neighbor shells of simulated water: Quantitative relation to translational and orientational order, Phys. Rev. E 76, in press.

[71] J. Wang, P. Cieplak, P.A. Kollman, J. Compd. Chem. 21 (2000) 1049.

[72] I. Brovchenko, A. Geiger, A. Oleinikova, J. Chem. Phys. 123 (2005) 044515.

[73] S.-H. Chen, L. Liu, X. Chu, Y. Zhang, E. Frattini, P. Baglioni, A. Faraone, E. Mamontov, J. Chem. Phys. XXX (2006) 171103.

[74] P. Kumar, Breakdown of the Stokes-Einstein relation in supercooled water, Proc. Natl. Acad. Sci. USA 103 (2006) $12955-12956$.

[75] P. Kumar, S.V. Buldyrev, S.L. Becker, P.H. Poole, F.W. Starr, H.E. Stanley, Relation between the Widom line and the breakdown of the Stokes-Einstein relation in supercooled water, Proc. Natl. Acad. Sci. 104 (2007) 9575-9579.

[76] P. Kumar, S.V. Buldyrev, H.E. Stanley, submitted.

[77] S.-H. Chen, F. Mallamace, C.-Y. Mou, M. Broccio, C. Corsaro, A. Faraone, The violation of the Stokes-Einstein relation in supercooled water, Proc. Natl. Acad. Sci. USA 103 (2006) 12974-12978.

[78] M.G. Mazza, N. Giovambattista, F.W. Starr, H.E. Stanley, Relation between rotational and translational dynamic heterogeneities in water, Phys. Rev. Lett. 96 (2006) 057803.

[79] M.G. Mazza, N. Giovambattista, H.E. Stanley, F.W. Starr, Dynamical heterogeneities and the breakdown of the Stokes-Einstein and Stokes-Einstein-Debye relations in simulated water, Phys. Rev. E 76 (2007) 031202.

[80] P. Kumar, G. Franzese, H.E. Stanley, Effects on the hydrogen bond network of crossing the Widom line, submitted.

[81] P. Kumar, S.V. Buldyrev, H.E. Stanley, Dynamic crossover and liquid-liquid critical point in the TIP5P model of water, in: S.J. Rzoska, V. Mazur (Eds.), Soft Matter under Extreme Pressures: Fundamentals and Emerging Technologies, Proceedings of the NATO ARW, Odessa, October 2005, Springer, Berlin, 2006.

[82] D. Liu, Y. Zhang, C.-C. Chen, C.-Y. Mou, P.H. Poole, S.-H. Chen, Observation of the density minimum in deeply supercooled confined water, Proc. Natl. Acad. Sci. 104 (2007) 9570-9574.

[83] D.A. Fuentevilla, M.A. Anisimov, Scaled equation of state for supercooled water near the liquid-liquid critical point, Phys. Rev. Lett. 97 (2006) 195702.

[84] T. Morishita, How does tetrahedral structure grow in liquid silicon on supercooling?, Phys. Rev. Lett. 97 (2006) 165502.

[85] F. Mallamace, M. Broccio, C. Corsaro, A. Faraone, D. Majolino, V. Venuti, L. Liu, C.-Y. Mou, S.H. Chen, Evidence of the existence of the low-density liquid phase in supercooled, confined water, Proc. Natl. Acad. Sci. 104 (2007) 424-428.

[86] F. Mallamace, S.-H. Chen, M. Broccio, C. Corsaro, V. Crupi, D. Majolino, V. Venuti, P. Baglioni, E. Fratini, C. Vannucci, H.E. Stanley, The role of the solvent in the dynamical transitions of proteins: the case of the lysozyme-water system, J. Chem. Phys. 127 (2007) 045104;

F. Mallamace, C. Branca, M. Broccio, C. Corsaro, N. Gonzalez-Segredo, H.E. Stanley, S.-H. Chen, Transport properties of supercooled confined water, Euro. Phys. J., submitted.

[87] H.E. Stanley, S.V. Buldyrev, M. Canpolat, O. Mishima, M.R. Sadr-Lahijany, A. Scala, F.W. Starr, The puzzling behavior of water at very low temperature, Phys. Chem. Chem. Phys. (PCCP) 2 (2000) 1551-1558 (Opening paper in Proc. International Meeting on Metastable Fluids, Bunsengesellschaft).

[88] O. Mishima, H.E. Stanley, The relationship between liquid, supercooled and glassy water, Nature 396 (1998) $329-335$. 
[89] H.E. Stanley, A polychromatic correlated-site percolation problem with possible relevance to the unusual behavior of supercooled $\mathrm{H}_{2} \mathrm{O}$ and $\mathrm{D}_{2} \mathrm{O}$, J. Phys. A 12 (1979) L329-L337.

[90] H.E. Stanley, J. Teixeira, Interpretation of the unusual behavior of $\mathrm{H}_{2} \mathrm{O}$ and $\mathrm{D}_{2} \mathrm{O}$ at low temperatures: tests of a percolation model, J. Chem. Phys. 73 (1980) 3404-3422.

[91] L. Bosio, J. Teixeira, H.E. Stanley, Enhanced density fluctuations in supercooled $\mathrm{H}_{2} \mathrm{O}, \mathrm{D}_{2} \mathrm{O}$, and ethanol-water solutions: evidence from small-angle X-ray scattering, Phys. Rev. Lett. 46 (1981) 597-600.

[92] M.C. Bellissent-Funel, Is there a liquid-liquid phase transition in supercooled water? Europhys. Lett. 42 (1998) $161-166$.

[93] J.M. Zanotti, M.-C. Bellissent-Funel, S.-H. Chen, Experimental evidence of a liquid-liquid transition in interfacial water, Europhys. Lett. 71 (2005) 91-97.

[94] J.M. Zanotti, M.-C. Bellissent-Funel, A.I. Kolesnikov, Phase transitions of interfacial water at 165 and 240 K. Connections to bulk water physics and protein dynamics, Euro. Phys. J. Special Topics 141 (2007) 227-233.

[95] J.M. Zanotti, M.-C. Bellissent-Funel, S.-H. Chen, et al., Further evidence of a liquid-liquid transition in interfacial water, J. Phys.-Cond. Mat. 18 (2006) S2299-S2304.

[96] S. Engemann, H. Reichert, H. Dosch, et al., Interfacial melting of ice in contact with $\mathrm{SiO}_{2}$, Phys. Rev. Lett. 92 (2004) 205701.

[97] F. Mallamace, C. Branca, M. Broccio, C. Corsaro, C.Y. Mou, S.H. Chen, Proc. Natl. Acad. Sci. USA, in press;

S.-H. Chen, et al. Dynamic Crossover Phenomenon in Confined Supercooled water and its relation to the existence of a liquid-liquid critical point in water, AIP Conf Proc., in press.

[98] U. Raviv, P. Laurat, J. Klein, Fluidity of water confined to subnanometre films, Nature 413 (2001) 51-54. 